\title{
Astrometric solar-system anomalies and evolutionary physical constants
}

\author{
Rajendra P. Gupta \\ Macronix Research Corporation, Ottawa, ON Canada K1J 8X4
}

\begin{abstract}
We have shown that three astrometric solar-system anomalies can be explained satisfactorily by using evolutionary gravitational constant $G$ and speed of light $c$ in the Einstein's field equation. These are: a) the Pioneer acceleration anomaly; b) the anomalous secular increase of Moon-orbit eccentricity; and c) the anomalous secular change in the astronomical unit AU. The gravitational constant $G$ and the speed of light $c$ both increase as $\mathrm{d} G / \mathrm{dt}=5.4 G H_{0}$ and $\mathrm{d} c / \mathrm{dt}=1.8 c H_{0}$ with $H_{0}$ as the Hubble constant. We also show that the Planck's constant $\hbar$ increases as $\mathrm{d} \hbar / \mathrm{dt}=1.8 \hbar H_{0}$. Additionally, the new approach fits the supernovae Ia redshift vs distance modulus data as well as the standard $\Lambda \mathrm{CDM}$ model with just one adjustable parameter $H_{0}$.
\end{abstract}

\section{INTRODUCTION}

The Pioneer anomaly originated from the work of Anderson et al. (1998) based on the radiometric data from Pioneer 10/11, Galileo, and Ulysses spacecraft that showed an apparent anomalous, constant acceleration acting on the spacecraft with a value of $\approx 8.5 \times$ $10^{-10} \mathrm{~m} \mathrm{~s}^{-2}$ directed towards the Sun. Anderson et al. (2002) improved this result to $(8.74 \pm 1.33) \times$ $10^{-10} \mathrm{~m} \mathrm{~s}^{-2}$ based on the additional analysis and modeling of the data. By taking into account certain thermal effects in the spacecraft, Feldman and Anderson (2015) reduced the anomaly to $7.69 \pm 1.15 \times$ $10^{-10} \mathrm{~m} \mathrm{~s}^{-2}$.

The Moon's orbit has an eccentricity that depends on the tidal forces due to surficial and the geophysical processes interior to the Earth and the Moon. After all the known sources responsible for the eccentricity $e$ were included, Williams and Dickey (2003) estimated that there remained a discrepancy of $\Delta \dot{e}=(16 \pm 5) \times$ $10^{-12} \mathrm{yr}^{-1}$ between the observed and calculated values. This value was revised downward by Williams and Boggs (2009) to $\Delta \dot{e}=(9 \pm 3) \times 10^{-12} \mathrm{yr}^{-1}$ and by Williams, Turyshev and Boggs (2014) to $\Delta \dot{e}=(5 \pm 2) \times$ $10^{-12} \mathrm{yr}^{-1}$ with updated data and tidal models.

Krasinsky and Brumberg (2004) from the analysis of all the radiometric measurements they had available between Earth and the major planets, which included observations of Martian landers and orbiters over the period of 1971 to 2003, determined a positive secular trend in Astronomical Unit (AU) estimated as $\frac{d A U}{d t}=$ $(15 \pm 4) \mathrm{m} \mathrm{cy}^{-1}$.

The astrometric solar system anomalies were reviewed and discussed in detail by Anderson and Nieto (2009), and more recently by Iorio (2015) along with other anomalies of the solar system. We will not therefore go into discussing the possible explanations offered by various researches for these anomalies. However, none of the explanations have been considered satisfactory.

Our approach to estimate the anomalies is based on the cosmological model that naturally and analytically yields the gravitational constant $G$ and speed of light $c$ that increase in time rather than decrease as believed by others (e.g. Dirac 1937, Barnes \& Dicke 1961). Together with the null results of Hofmann and Muller's (2018) attempts to determine the variation of gravitational constant $G$ by analysing several decades of LLR data, the new model yields $\dot{G} / G=5.4 H_{0}$ and $\dot{c} / c=1.8 H_{0}$. These are the only two parameter (and of course the Hubble constant $H_{0}$ ) required for estimating all the three anomalies. However, before we do that let us see how we get $\dot{G} / G$ and $\dot{c} / c$.

\section{EVOLUTIONARY CONSTANTS MODEL}

We will develop our model in the general relativistic domain starting from the Robertson-Walker metric with the usual coordinates $x^{\mu}(c t, r, \theta, \phi)$ :

$d s^{2}=c^{2} d t^{2}-a(t)^{2}\left[\frac{d r^{2}}{1-k r^{2}}+r^{2}\left(d \theta^{2}+\sin ^{2} \theta d \phi^{2}\right)\right] .(1)$

Here $a(t)$ is the scale factor and $k$ determines the spatial geometry of the universe: $k=-1$ (closed), 0 (flat), +1 (open). The Einstein field equations may be written in terms of the Einstein tensor $G^{\mu \nu}$, metric tensor $g^{\mu \nu}$, energymomentum tensor $T^{\mu \nu}$, cosmological constant $\Lambda$, gravitational constant $G$ and speed of light $c$, as:

$G^{\mu \nu}+\Lambda g^{\mu \nu}=-\frac{8 \pi G}{c^{4}} T^{\mu \nu}$.

When solved for the Robertson-Walker metric, we get the following non-trivial equations for the flat universe $(k=0)$ 


\section{R. P. Gupta}

Astrometric solar-system anomalies 2 of interest to us here, with $p$ as the pressure and $\varepsilon$ as the energy density:

$\frac{\ddot{a}}{a}+\frac{1}{2}\left(\frac{\dot{a}}{a}\right)^{2}=-\frac{4 \pi G}{c^{2}} p+\frac{1}{2} \Lambda$

$\frac{\dot{a}^{2}}{a^{2}}=\frac{8 \pi G}{3 c^{2}} \varepsilon+\frac{1}{3} \Lambda$

If we do not regard $G, c$ and $\Lambda$ to be constant and define $K \equiv \frac{G}{c^{2}}$, we may easily derive the continuity equation by taking time derivative of Eq. (4) and substituting in Eq. (3)

$\dot{\varepsilon}+\frac{3 \dot{a}}{a}(\varepsilon+p)+\frac{\dot{K}}{K} \varepsilon+\frac{\dot{\Lambda}}{8 \pi K}=0$.

It reduces to the standard continuity equation when $K$ and $\Lambda$ are held constant. And since the Einstein field equations require that the covariant derivative of the energymomentum tensor $T^{\mu \nu}$ be zero, we can interpret Equation (5) as comprising of two continuity equations (Maharaj \& Naidoo 1993), viz

$\dot{\varepsilon}+\frac{3 \dot{a}}{a}(\varepsilon+p)=0$, and

$8 \pi \varepsilon \dot{K}+\dot{\Lambda}=0$.

This separation simplifies the solution of the field equations (Eqs. 3 and 4). Eq. (6) yields the standard solution for the energy density $\varepsilon=\varepsilon_{0} a^{-3(1+w)}$. Here $w$ is the equation of state parameter defined as $p \equiv w \varepsilon$ with $w=0$ for matter, $1 / 3$ for radiation and -1 for $\Lambda$.

As has been explicitly delineated by Magueijo in several of his papers (e.g. Magueijo 2000), this approach is not generally Lorentz invariant. Nonetheless it remains relativistic and locally Lorentz invariant.

Since the universe expansion is determined by $H(t) \equiv$ $\dot{a} / a$, it is natural to assume the time dependence of any time dependent parameter to be proportional to $\dot{a} / a$ (the so called Machian scenario - Magueijo 2003). Let us therefore write

$\frac{\dot{K}}{K}=k\left(\frac{\dot{a}}{a}\right), \frac{\dot{\Lambda}}{\Lambda}=l\left(\frac{\dot{a}}{a}\right)$ and $\frac{\dot{H}}{H}=m\left(\frac{\dot{a}}{a}\right)$, i.e.

$K=K_{0} a^{k}, \Lambda=\Lambda_{0} a^{l}$ and $H=H_{0} a^{m}$.

Here $k$ (not the same as in Eq. (1)) $l$ and $m$ are the proportionality constants, and subscript zero indicates the parameter value at present $\left(t=t_{0}\right)$. With this substitution in Eq. (4) we may write

$\frac{\dot{a}^{2}}{a^{2}}=H_{0}^{2} a^{2 m}=\frac{8 \pi}{3}\left(K_{0} a^{k}\right) \varepsilon_{0} a^{-3(1+w)}+\frac{1}{3} \Lambda_{0} a^{l}$.

Comparing the exponents of $a$ of all the terms, we may write $2 m=k-3-3 w=l$, and with $w=0$ for matter, we have $2 m=k-3=l$. Thus, if we know $k$, we know $l$ and $m$.

We can now have a closed analytical solution of Eq. (10) as follows (since $a\left(t_{0}\right) \equiv 1$ ):

$a(t)=\frac{a(t)}{a\left(t_{0}\right)}=\left(\frac{t}{t_{0}}\right)^{\frac{2}{3+3 w-k}} ; \frac{\dot{a}}{a}=\frac{2}{3+3 w-k} t^{-1} ;$ $\frac{\ddot{a}}{\dot{a}}=\left(\frac{\dot{a}}{a}\right)\left(1-\frac{3+3 w-k}{2}\right) ;$ and $-q \equiv \frac{\ddot{a} a}{\dot{a}^{2}}=\frac{-1-3 w+k}{2}$.

Here $q$ is deceleration parameter. It may be noticed that $q$ does not depend on time, i.e. $q_{0}=q$. As we know the radiation energy density is negligible at present, so we need to be concerned with the matter only solutions, i.e. with $w=0$.

The deceleration parameter $q_{0}$ has been analytically determined on the premise that expansion of the universe and the tired light phenomena are jointly responsible for the observed redshift, especially in the limit of very low redshift (Gupta 2018). One could see it as if the tired light effect is superimposed on the Einstein de Sitter's matter only universe rather than the cosmological constant. By equating the expressions for the proper distance of the source of the redshift for the two, one gets $q_{0}=-0.4$. Then from Eq. (12) we get $k=1.8$, and also $l=$ -1.2 , and $m=-0.6$. We thus have from Eq. (8) $\dot{K} / K=$ $1.8 H, \dot{\Lambda} / \Lambda=-1.2 H$ and $\dot{H} / H=-0.6 H$.

\section{VARYING $\boldsymbol{G}$ AND $\boldsymbol{c}$ FORMULATION}

Having determined the value of $k=1.8$, and since the Hubble parameter is defined as $H=\dot{a} / a$, we may write from Eqs. (8) and (9)

$K=K_{0} a^{1.8}$, and $\frac{\dot{K}}{K}=1.8 H$.

We may also write explicitly

$\frac{\dot{K}}{K}=\frac{\dot{G}}{G}-\frac{2 \dot{C}}{c}=1.8 H$.

Taking $H$ at the present time as $H_{0} \simeq 70 \mathrm{~km} \mathrm{~s}^{-1} \mathrm{Mpc}^{-1}$ $\left(2.27 \times 10^{-18} \mathrm{~s}^{-1}\right)$ we get $\frac{\dot{K}}{K}=4.09 \times 10^{-18} \mathrm{~s}^{-1}=1.29$ $\times 10^{-10} \mathrm{yr}^{-1}$.

The findings from the Lunar Laser Ranging (LLR) data analysis provides the limits on the variation of $\dot{G} / G$ $\left(7.1 \pm 7.6 \times 10^{-14}\right)$ (Hofmann \& Müller 2018), which is considered to be about three orders of magnitude lower than expected (Dirac 1937, Barnes \& Dicke 1961). However, the LLR data analysis is based on the assumption that the speed of light is constant and non-evolutionary. If this constraint is dropped then the finding would be very different.

As is well known (Merkowitz 2010), a time variation of $G$ should show up as an anomalous evolution of the orbital period $P$ of astronomical bodies expressed by Kepler's 3rd law:

$P^{2}=\frac{4 \pi^{2} r^{3}}{G M}$,

where $r$ is semi-major axis of the orbit, $G$ is the gravitational constant and $M$ is the mass of the bodies involved in the orbital motion considered. If we take time derivative of Eq. (15), divide by $P^{2}$ and rearrange, we get

$\frac{\dot{G}}{G}=\frac{3 \dot{r}}{r}-\frac{2 \dot{P}}{P}-\frac{\dot{M}}{M}$. 


\section{R. P. Gupta}

Astrometric solar-system anomalies 3

If we write $r=c t$ then $\frac{\dot{r}}{r}=\frac{1}{t}+\frac{\dot{c}}{c}$. We may now rewrite Eq. (16) as

$\frac{\dot{G}}{G}-\frac{3 \dot{C}}{c}=\frac{3}{t}-\frac{2 \dot{P}}{P}-\frac{\dot{M}}{M}$.

Since LLR measures the time of flight of the laser photons, it is the right hand side of Eq. (17) that is determined from LLR data analysis (Hofmann \& Müller 2018) to be $7.1 \pm 7.6 \times 10^{-14}$ and not the right hand side of Eq. (16).

Then, taking the right hand side of Eq. (17) as 0 and combining it with Eq. (14), one can solve the two equations and get $\dot{G} / G=5.4 H$ and $\dot{c} / c=1.8 H$. It should be emphasized that both $\dot{G} / G$ and $\dot{c} / c$ are positive and thus both of them are increasing with time rather than decreasing as is generally believed (e.g. Dirac 1937, van Flandern 1975). This may be considered the most significant observational finding of cosmological consequences just by studying the Earth-Moon system.

\section{PIONEER ANOMALY}

Having determined the values of $\dot{G} / G$ and $\dot{c} / c$ we can now proceed to calculate the anomalous acceleration towards the Sun of Pioneer 10 and 11 spacecraft (Feldman $\&$ Anderson 2015). Since gravitational pull of Sun on the spacecraft decreases according the inverse square law, $\frac{\dot{G}}{G}$ cannot be expected to give a constant acceleration independent of the distance of the spacecraft. If the acceleration is denoted by $f$, one can easily workout using the Newtonian relation $f=G M / r^{2}$, that $\dot{f} / f=-3.74 \times$ $10^{-19} \mathrm{~s}^{-1}$, which yields negligible anomalous acceleration. Thus, we need to only consider the effect of $\dot{c} / c$ from a different perspective. If the spacecraft is at a distance $r_{0}$ from Earth then the signal from Earth will have a two way transit time $\Delta t$ given by $2 r_{0}=c_{0} \Delta t$ assuming $c_{0}$ as the speed of light. But, if the speed of light is evolving as $\dot{c} / c=1.8 H_{0}$ near $t=t_{0}$, i.e. as $c=c_{0} e^{1.8 H_{0}\left(t-t_{0}\right)}$ during the transit time then the actual transit time will be shorter than $\Delta t$ (since $c>c_{0}$ for $t>t_{0}$ ). Because of the shorter actual transit time, an observer would consider the spacecraft to be nearer to Earth than it actually is and thus would think that there is a deceleration of the spacecraft due to some unaccounted cause.

We could write the proper distance of the spacecraft $r_{p}$ and its apparent distance $r_{a}$ as:

$2 r_{a}=c_{0} \Delta t$, and

$2 r_{p}=c_{0} \int_{0}^{\Delta t} e^{1.8 H_{0} t} d t=\frac{c_{0}}{1.8 H_{0}}\left(e^{1.8 H_{0} \Delta t}-1\right)$,

and since $1.8 H_{0} \Delta t \ll 1$,

$2 r_{p}=\frac{c_{0}}{1.8 H_{0}}\left[\left(1+1.8 H_{0} \Delta t+\frac{1}{2}\left(1.8 H_{0}\right)^{2} \Delta t^{2} \ldots.\right)-1\right]$, or

$r_{p}=\frac{1}{2} c_{0} \Delta t+\frac{1.8 H_{0}}{4} c_{0} \Delta t^{2}=r_{a}+\frac{1}{2}\left(0.9 H_{0} c_{0}\right) \Delta t^{2}$, or

$r_{a}=r_{p}-\frac{1}{2}\left(6.129 \times 10^{-10} \mathrm{~m} \mathrm{~s}^{-2}\right) \Delta t^{2}$.
Thus the acceleration is $-6.129 \times 10^{-10} \mathrm{~m} \mathrm{~s}^{-2}$, and since it is negative, it is towards the observer at Earth.

Out of $\mathbf{7 . 6 9} \pm \mathbf{1 . 1 7} \times \mathbf{1 0}^{-10} \mathrm{~m} \quad \mathrm{~s}^{-2}$ anomalous acceleration of Pioneer 10 and 11 towards the Sun (truly towards Earth) we are able to analytically account for $6.13 \times 10^{-10} \mathrm{~m} \mathrm{~s}^{-2}$, leaving only $1.56 \pm 1.17 \times 10^{-10}$ $\mathrm{m} \mathrm{s}^{-2}$ as the anomaly.

It should be mentioned that Kopeikin (2012) has obtained essentially the same result and explained it as due to the cosmological effect of quadratic divergence between the electromagnetic and atomic time scales governing the propagation of radio wave in the Doppler tracking system and the atomic clock on Earth respectively. However, his approach is not conducive to explaining the other two anomalies.

\section{MOON'S ECCENTRICITY ANOMALY}

The eccentricity $e$ of the orbit of Moon may be written as (Bates et al. 1971)

$e=\sqrt{1+\frac{2 \epsilon h^{2}}{\mu^{2}}}$, or $e^{2}-1=\frac{2 \epsilon h^{2}}{\mu^{2}}$.

Here $\epsilon=-\mu / 2 a_{m}$ is the specific orbital energy, $\mu=$ $G\left(m_{e}+m_{m}\right)$ is the gravitational parameter for Earth-Moon system, $\boldsymbol{h}=\boldsymbol{r} \times \boldsymbol{v} m_{e} / M_{r}$ is the specific relative angular momentum, $a_{m}$ is the semi-major axis of the orbit, $m_{e}$ is mass of the Earth, $m_{m}$ is the mass of the Moon, $\boldsymbol{r}$ is the radius vector and $\boldsymbol{v}$ is the velocity vector of the Moon, and $M_{r}=m_{e} m_{m} /\left(m_{e}+m_{m}\right)$ is the reduced mass. Taking $a_{m}=r$ and assuming $\boldsymbol{r}$ is normal to $\boldsymbol{v}$, we may write Eq. (20) as

$1-e^{2}=\frac{r v^{2}}{G}\left(\frac{m_{e}+m_{m}}{m_{e}^{2}}\right)$.

Differentiating this equation with respect to time, assuming the mass factor to be constant, and dividing by the same equation, we get

$-\frac{e \dot{e}}{1-e^{2}}=\frac{\dot{r}}{r}+\frac{2 \dot{v}}{v}-\frac{\dot{G}}{G}$, and since $e \ll 1$,

$e \dot{e}=\frac{\dot{G}}{G}-\frac{\dot{r}}{r}-\frac{2 \dot{v}}{v}$.

Since $r$ is measured optically, the speed of light enters in it, i.e. $r=c t$, or $\dot{r}=v=\dot{c} t+c \dot{t}$, or $\frac{\dot{r}}{r}=\frac{\dot{c}}{c}+\frac{1}{t}$. Since all the parameters are expressed at current time, $t$ in the denominator must be expressed in terms of the Hubble time $1 / H_{0}$. However, it is better to write $t=p H_{0}^{-1}$ where $(1-p)$ is the small factor very close to 0 that may be considered to correct for the approximations made in our model ( $p$ here is not pressure). We can also determine $\frac{\dot{v}}{v}$ : $\dot{v}=\ddot{r}=\ddot{c} t+\dot{c}+\dot{c}=2 \dot{c}$, assuming $\dot{c}$ as constant. Thus $\frac{\dot{v}}{v}=\frac{2 \dot{c}}{c t+c}=\frac{2 \dot{c}}{c} /\left(\frac{\dot{c}}{c} t+1\right)$. We may therefore write Eq. (23) as

$e \dot{e}=\frac{\dot{G}}{G}-\left(\frac{\dot{c}}{c}+\frac{1}{t}\right)-\frac{4 \dot{c}}{c} /\left(\frac{\dot{c}}{c} t+1\right)$, or 


\section{R. P. Gupta}

Astrometric solar-system anomalies 4

$\frac{e \dot{e}}{H_{0}}=5.4-\left(1.8+\frac{1}{p}\right)-\frac{7.2}{1.8 p+1}$.

For $\quad p=1, e \dot{e} / H_{0}=0.0285715$. And taking $H_{0}=$ $0.716 \times 10^{-10} \mathrm{yr}^{-1}$, and $e=0.0549$ for Moon, we get $\dot{e}=37 \times 10^{-12} \mathrm{yr}^{-1}$. This is about twice the original value of the anomalous rate of eccentricity increase

The value determined is very sensitive to the value of the parameter $p$. It can be approximated near $p=1$ with an expression:

$\frac{e \dot{e}}{H_{0}}=0.0285714+2.65306(p-1)$.

There are three values of $\dot{e}$ that are significant here:

a) $\dot{e}=16 \pm 5 \times 10^{-12} \mathrm{yr}^{-1}$ originally estimated (Williams \& Dickey 2003); it gives $p=0.993855 \pm$ 0.001445 .

b) $\dot{e}=9 \pm 3 \times 10^{-12} \mathrm{yr}^{-1}$ updated value using more data and 'better' tidal effect model (Williams and Boggs 2009); it gives $p=0.991832 \pm 0.000867$.

c) $\dot{e}=5 \pm 2 \times 10^{-12} \mathrm{yr}^{-1}$ updated value with even more data and 'even better' tidal effect model (Williams et al. 2014); it gives $p=0.990676 \pm 0.000578$.

All the values of $p$ are very close to 1 , indicating that our model is very good approximation to the exact solution of the Einstein field equations, at least locally, with variable $c$ and $G$.

\section{AU ANOMALY}

The orbit of Earth around Sun is Keplerian and thus is governed by Eq. (15). A radiometric measurement will therefore yield a null result in our approach using evolutionary $G$ and $c$. However, the eccentricity evolution is equally valid for the Moon and the Earth. Thus applying Eq. (25) to the Earth's orbit, and assuming $p=1$ and $e=$ 0.0167 , we get $\dot{e}=122.5 \times 10^{-12} \mathrm{yr}^{-1}$. This can be translated easily into AU increase as follows. The semimajor axis $a$ and semi-minor axis $b$ of an orbit may be written as (Bates et al. 1971)

$a=\frac{p_{s}}{1-e^{2}}$, and $b=\frac{p_{s}}{\sqrt{1-e^{2}}}$.

Here, $p_{s}=a=b$ defines a circle when $e=1$. AU may then be written as

$$
\begin{aligned}
A U=\frac{a+b}{2}= & \frac{p_{s}}{2}\left(\frac{1}{1-e^{2}}+\frac{1}{\sqrt{1-e^{2}}}\right), \\
& =p_{s}\left(1+\frac{3}{4} e^{2}+\frac{11}{16} e^{4}+O\left(e^{6}\right)\right) .
\end{aligned}
$$

Suppose now that the eccentricity $e$ increases by $\Delta e$ to $e^{\prime}$ in a time period $\Delta t$. Then $e^{\prime}=e+\Delta e$ and $e^{\prime 2}=e^{2}+2 e^{2} \Delta e$ when we ignore higher order terms in $\Delta e$. We may now write the increase in $\mathrm{AU}$ as $\triangle \mathrm{AU}$ :

$$
\Delta A U=\frac{3}{2} p_{s} e^{2} \Delta e, \text { or } \frac{d A U}{d t}=\frac{3}{2} e^{2} \dot{e} \times A U .
$$

Here we have approximated $p_{s}=A U$ since $e^{2} \ll 1$. Taking $A U=1.496 \times 10^{11} \mathrm{~m}$ and using Eq. (25) for $e \dot{e}$ with $p=1$, we get $d A U / d t=0.77 \mathrm{~m} \mathrm{cy}^{-1}$ against its measured value of $1.5 \mathrm{~m} \mathrm{cy}^{-1}$. As can be seen from Eq. (26), if we took $p=1.010$ instead of 1 , we would get the desired value. The reason could be the same as discussed at the end of previous section. Alternatively, there may be other phenomena contributing to the anomalous AU.

It should be mentioned that recently the $\mathrm{AU}$ has been redefined (e.g. Capitaine 2012) and just by definition the AU anomaly has been eliminated. One has to resort to the old definition of AU to appreciate the AU anomaly and its resolution.

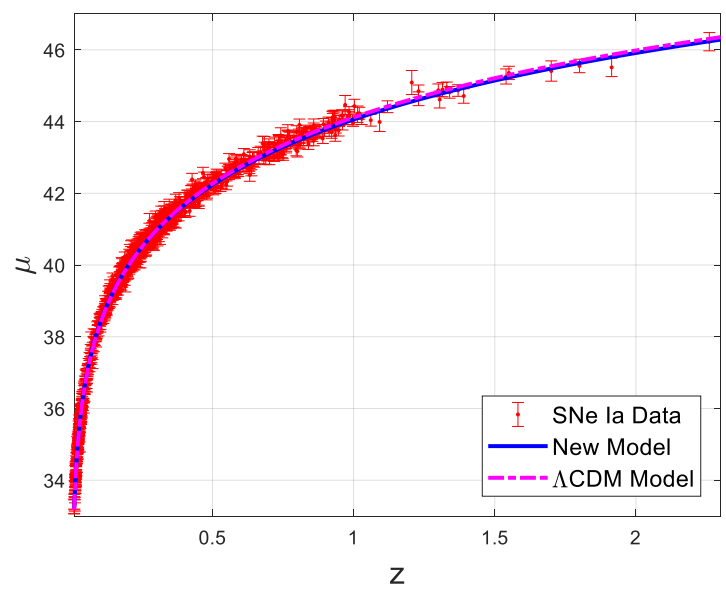

FIG. 1. Supernovae Ia redshift $z$ vs distance modulus $\mu$ data fit based on the model developed in Sec. II as compared to the fit using the $\Lambda \mathrm{CDM}$ model.

\section{SUPERNOVAE Ia $z-\mu$ DATA FIT}

We tried the new model developed here to see how well it fits the best supernovae Ia data (Scolnic et al. 2018)) as compared to the standard $\Lambda \mathrm{CDM}$ model. The data fit is shown in Fig. 1. The new model requires only one parameter to fit the data $\left(H_{0}=68.92 \pm 0.26 \mathrm{~km}\right.$ $s^{-1} \mathrm{Mpc}^{-1}$ ) whereas the $\Lambda \mathrm{CDM}$ model requires two parameters $\left(H_{0}=70.18 \pm 0.43 \mathrm{~km} \mathrm{~s}^{-1} \mathrm{Mpc}^{-1}\right.$ and $\Omega_{m, 0}=$ $0.2854 \pm 0.0245$ with $\Omega_{m, 0}$ as the matter energy density relative to the total energy density). The excellent goodness-of-fit provides added confidence in the new approach for its cosmological relevance. The details of the work are beyond the scope of this short paper.

\section{VARIATION OF PLANCK'S CONSTANT h}

The variation of the fine structure constant $\alpha=$ $\left(1 / 4 \pi \epsilon_{0}\right) \mathrm{e}^{2} / \mathrm{h} c$ (here $\epsilon_{0}$ is the vacuum permittivity and $\mathrm{e}$ is electron charge) has been studied extensively. Since $\epsilon_{0}=1 / \mu_{0} c^{2}$, where $\mu_{0}$ is the vacuum permeability, $\alpha=\left(\mu_{0} / 4 \pi\right) e^{2} c / \hbar$. Recent estimates put a very low value on $\dot{\alpha}$ (Gohar 2017, Rosenband et al. 2008). We may write $\frac{\dot{\alpha}}{\alpha}=2 \frac{\dot{\mathrm{e}}}{\mathrm{e}}-\frac{\dot{\hbar}}{\hbar}+\frac{\dot{c}}{c}$. If $\dot{\alpha} / \alpha$ and $\dot{\mathrm{e}} / \mathrm{e}$ are zero, or varying very 


\section{R. P. Gupta}

Astrometric solar-system anomalies 5

little compared to $\dot{\hbar} / \hbar$ and $\dot{c} / c$, and $\mu_{0}$ is a constant, then it is implied that $\dot{\hbar} / \hbar=\dot{c} / c=1.8 H$.

\section{CONCLUSION}

We wish to make several points:

1. One could see that the approach taken here to explain the three anomalies is based on a very simple analytically derived expression for the evolution of the speed of light and gravitational constant. Thus one could infer that the Occam's razor principle would favour the new approach over other approaches. In the case of the Pioneer anomaly, it should be rather easy to implement it in the real time modeling of the astrodynamics of long range spacecraft.

2. The expression of eccentricity variation involves $G$ and $c$ in a manner that the contribution of $\dot{G} / G$ and $\dot{c} / c$ almost cancels each other except for a small residual. This may be compared with the case of using Kepler's Eq. (15) when the two contributions cancel out entirely.

3. Based on $G$ variation alone, one can see from Eqs. (23-25) that calculated $\dot{e}$ is more than two orders of magnitude higher than measured $\dot{e}$. Current work corrects this by including $\dot{c}$ variation.

4. There may be bias factors in the models (say in favour of tidal effects) used for data analysis since a model is not considered good enough unless it can account for all the observed value. We believe that one may be able to remove this bias by the inclusion of local effect of cosmology, as presented here, in the data analysis models.

5. As mentioned above both $\dot{G} / G$ and $\dot{c} / c$ are positive and thus both of them are increasing with time rather than decreasing. The simple model presented above effectively eliminates the need for the cosmological constant. The existence of cosmological constant $\Lambda$ in Friedmann equation leads to a continuous addition of dark energy to the universe as the universe expands, i.e. it causes the total energy of the universe to increase. The same is achieved by the increase of $G$ and $c$ through the second continuity equation (Eq. 7).

6. Variability of all the constants is expressed in terms of the Hubble parameter $H(t)$, and at the present time relative to the Hubble constant $H_{0}$. In summary the physical constants evolve as follows: $\dot{c} / c=1.8 H, \dot{G} / G=$ $5.4 H, \dot{\hbar} / \hbar=1.8 H, \dot{\Lambda} / \Lambda=-1.2 H$, and $\dot{H} / H=-0.6 H$.

\section{ACKNOWLEDGEMENT}

Author is thankful to Franz Hofmann for providing his latest research paper and references on Lunar Laser Ranging. He is grateful to Dan Scolnic for providing the latest supernovae Ia data.

\section{REFERENCES}

Anderson, J. D., Laing, P. A., Lau, E. L., Liu, A. S., Nieto, M. M., Turyshev, S. G., 1998, Phys. Rev. Lett. $81,2858$.

Anderson, J. D., Laing, P. A., Lau, E. L., Liu, A. S., Nieto, M. M., Turyshev, S. G., 2002, Phys. Rev. D 65, 082004.

Anderson, J. D., Nieto, M. M., 2009 Relativity in Fundamental Astronomy: Dynamics, Reference Frames, and Data Analysis Proceedings, eds. S. Klioner, P. K. Seidelmann and M.Soffel, IAU Symposium No. 261, 1.

Barnes, C., Dicke, R. H., 1961 Phys. Rev. 124, 925.

Bates, R. R., Mueller, D. D., White, J. E., 1971, Fundamentals of Astrodynamics (Dover Publication, New York).

Capitaine, N., 2012, Proceedings of the "Journées 2011 Systèmes de référence spatio-temporels", eds. H. Schuh, S. Bo"hm, T. Nilsson and N. Capitaine (Vienna University of Technology,), pp. 266-269.

Dirac, P. A. M., 1937, Nature 139, 323.

Feldman, R. M., Anderson, J. D., 2015, Int'1 J. Mod. Phys. 24, 1550066.

Gohar, H., (2017) Universe 3, 26.

Gupta, R. P., 2018 Int'1 J. Astron. Astrophys. 8, 219.

Hofmann, F., Müller, J., 2018, Class. Quant. Grav. 35, 035015.

Iorio, L., (2015) Int'l J. Mod. Phys. D 24, 1530015.

Kopeikin, S. M., 2012, Phys. Rev. D 86, 064004.

Krasinski, S. M., Brumberg, V. A., 2004 Cel. Mech. Dyn. Astron. 90, 267.

Magueijo, J., 2000 Phys. Rev. D 62, 103521.

Magueijo, J., 2003, Rept. Prog. Phys. 66, 2025.

Maharaj, S. D., Naidoo, R., 1993 Astrophys. Sp. Sci. 208, 261.

Rosenband, T., Hume, D. B., Schmidt, P. O., et al., 2008, Science 319, 1808.

Scolnic, D. M., Jones, D. O., Rest, A., et al., 2018 Astrophys. J. 859, 101; data file taken on Dec. 11, 2018 from Catalogs of Cosmologically Useful Type Ia Supernovae from Pan-STARRS ("PS1COSMO") at https://archive.stsci.edu/hlsps/ps1cosmo/scolnic/hlsp_ps1 cosmo_panstarrs_gpc1_all_model_v1_lcparam-full.txt.

van Flandern, T. C., 1975 Mon. Not. Roy. Astron. Soc. $170,333$.

Williams, J. G., Dickey, J. O., 2003, Proceedings of the $13^{\text {th }}$ International Workshop on Laser Ranging, Eds. R. Noonan, S. Klosko, C. Noll and M. Pearlman, NASA/CP-2003-212248, pp.1-12.

Williams, J. G., Boggs, D. H., 2009 Proceedings of the 16th International Workshop on Laser Ranging October 2008, Pozna'n, Poland, ed. S. Schillak (Space Research Centre of the Polish Academy of Sciences, Warszawa), pp. 101-120.

Williams, J. G, S. G. Turyshev, S. G., Boggs, D. H., 2014, Planet. Sci. 3, 2. 